\title{
Photonic Crystal Lasers
}

\author{
Axel Scherer, Oskar Painter, Zhaoyu Zhang, Yueming Qiu ${ }^{1}$ Tomo Yoshie ${ }^{2}$ \\ Caltech, MC 200-36 Caltech, Pasadena CA 91125 \\ 1: Jet Propulsion Laboratory \\ 2: Duke University
}

\begin{abstract}
We compare photonic crystal lasers with other microcavities for future switching and spectroscopy systems. High speed and efficiency along with massive lithographic integration makes these lasers particularly interesting for optical switching and signal processing
\end{abstract}

Keywords: Photonic Crystals, Lasers, Microcavities

\section{Introduction}

Over the past several years, we have developed lithography and etching approaches to define optical microcavities with small mode volumes. When gain is added to these optical cavities, it is possible to define small lasers, such as photonic crystal lasers, microdisk lasers, vertical cavity lasers, annular Bragg reflector lasers, and folded cavity lasers. Such lasers have become very interesting for applications in spectroscopy (Figure 1) and
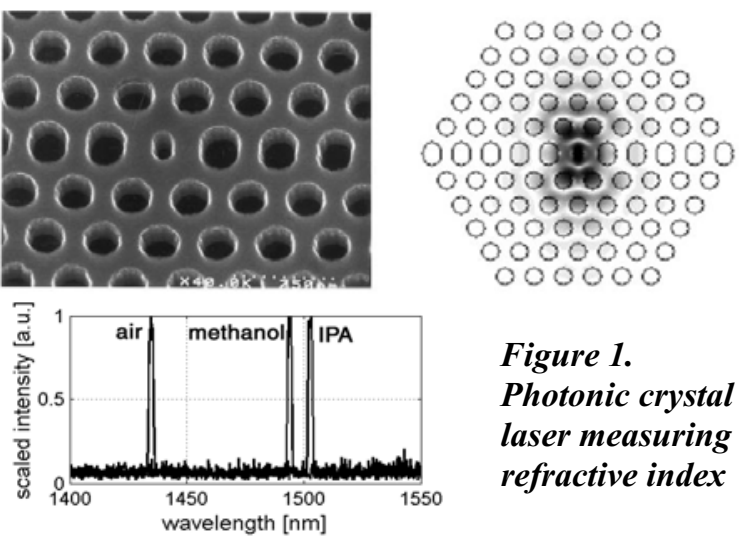

Figure 1.

Photonic crystal

laser measuring

refractive index

fast switching. In this presentation, we compare these geometries and describe applications of these devices in future switching and spectroscopy systems. The potential for high speed and efficiency as well as massive lithographic integration makes photonic crystal laser geometries particularly interesting for optical switching and signal processing applications.

\section{Opportunities offered by Small Mode Volumes}

A key advantage of reducing the mode volume of lasers lies in an increased Purcell factor and the opportunity to increase the switching speed within these optical cavities. When the quantum well active material is replaced with quantum dot material, it is also possible to define solid state systems in which strong coupling between the quantum dots and the high- $Q$ optical cavities can be observed. We have also defined quantum dot lasers and coupled cavity laser arrays in which the more dilute quantum dot material can be used to reduce re-absorption losses within the lasers. Quantum dot photonic crystal devices are expected to lead to several new applications including very efficient light sources, ultra-small electrooptic modulators and highly sensitive detectors. The integration of lithographically defined devices embedded within photonic crystal material also enables many optical cavities to be connected in complex multi-wavelength systems.

\section{Photonic Crystal Laser Sensors}

These small and highly efficient light sources have also recently been integrated with microfluidic sample delivery systems (Figure 2) to define tunable spectroscopic systems. Most of the microfluidic structures are fabricated by replication molding from polydimethyl-siloxane elastomer and can be aligned with the spectroscopic light sources. By integrating the fluidic

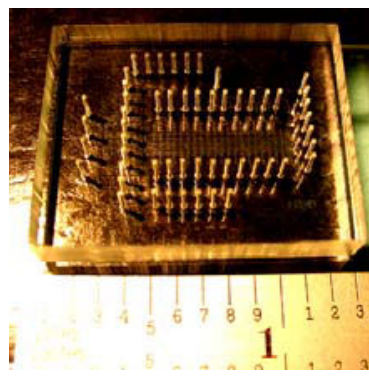

Figure 2. Fluidic chip for sample delivery to lasers with photonic devices, we have demonstrated rapid pico-Liter sample delivery to sensors with femto-Liter analytical volumes. In general, such device miniaturization results in the opportunity to reduce the sample volumes and is often accompanied by significant improvements in the sensitivity and speed of the analysis. Recently, it has become possible to define these lasers in InGaP materials emitting within the visible wavelength range, making many new chemical and
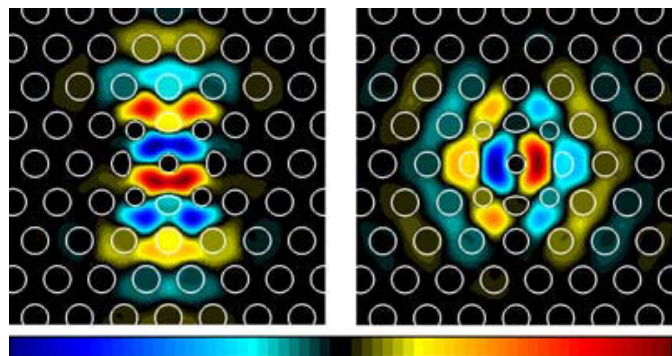

Figure 3. Q-switched modes of Photonic crystal laser when backfilled with birefringent nonlinear polymer. 
biological analysis applications possible. Conversely, the lasers can also be tuned through the addition of polymers that can change in refractive index through second or third order nonlinear effects.

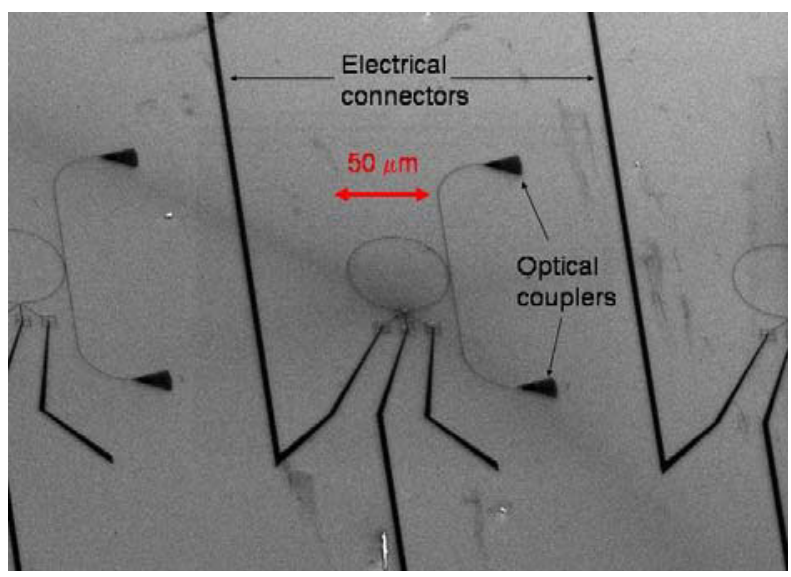

Figure 4. Silicon photonic split ring resonators serving as tunable add/drop filters

Systems based on index-guided silicon on insulator technologies (Figure 4) will also be compared with photonic crystal devices. The introduction of gain into spectroscopic systems will be introduced, in particular with respect to the use of the addition of dyes and electrooptic polymers to define opto-fluidic laser sources. The introduction of nonlinear polymers within these devices can be used to tune and switch these lasers efficiently, and we will describe these emerging devices.

\section{Conclusions}

Clearly, many opportunities exist for lithographically defined optical systems to generate, confine, guide and switch light for high frequencies communication and sensing applications. Photonic crystal lasers may well fill an important role in the definition of such highly integrated photonic systems. Applications of photonic crystal chips presently range from quantum optics to biochemical sensing, and eventually may enable dense optical switch networks to be fabricated.

\section{Acknowledgments}

The authors gratefully acknowledge the generous support from the AFOSR under program F49620-03-1-0418 and from the Boeing corporation under the SRDMA program. 